\title{
miR-200a-3p promotes the proliferation of human esophageal cancer cells by post-transcriptionally regulating cytoplasmic collapsin response mediator protein-1
}

\author{
YANZI ZANG ${ }^{*}$, YONG TAI ${ }^{*}$, BAOLUO WAN and XIAODONG JIA \\ Department of Otolaryngology, People's Hospital of Henan Province, Zhengzhou, Henan 450003, P.R. China
}

Received November 29, 2015; Accepted August 12, 2016

DOI: $10.3892 /$ ijmm.2016.2758

\begin{abstract}
The dysregulation of cytoplasmic collapsin response mediator protein 1 (CRMP1) has been reported in lung cancer, medulloblastoma and esophageal cancer. However, the role of CRMP1 and its regulatory mechanisms in esophageal cancer remain unclear. In this study, we demonstrated that CRMP1 expression was downregulated in esophageal cancer tissues and that there were differences in its expression levels in different esophageal cancer cell lines. We found that CRMP1 overexpression inhibited the proliferation of esophageal cancer cells, whereas the silencing of CRMP1 promoted cell proliferation. We performed an analysis of potential microRNA (miRNA or miR) target sites using a commonly used prediction algorithm (TargetScan). The algorithm predicted that miR-200a-3p targets the 3' untranslated region (3'UTR) of CRMP1. Further experiments confirmed this prediction. In addition, we found that miR-200a-3p promoted the proliferation of esophageal cancer cells. Thus, our findings indicate that miR-200a-3p promotes the proliferation of human esophageal cancer cells by post-transcriptionally regulating CRMP1.
\end{abstract}

\section{Introduction}

Esophageal cancer (EC) is one of the six most common malignancies worldwide, with a higher incidence in males than in females (1). The lifetime risk of developing this type of cancer is $0.8 \%$ for men and $0.3 \%$ for women. In addition, the risk increases with age, with a mean age at diagnosis of 67 years $(2,3)$. $\mathrm{EC}$ is the seventh leading cause of cancer-related mortality among American males, particularly African American males,

Correspondence to: Dr Yanzi Zang, Department of Otolaryngology, People's Hospital of Henan Province, 7 Weiwu Road, Zhengzhou, Henan 450003, P.R. China

E-mail: zangyanzi001@126.com

${ }^{*}$ Contributed equally

Key words: esophageal cancer, cytoplasmic collapsin response mediator protein 1 , miR-200a-3p who have a higher incidence of this disease (13 cases per 100,000 individuals) than do males of other racial or ethnic origin $(2,4)$. Elucidating molecular alterations in EC will help us to find new targets for effective therapies.

Cytoplasmic collapsin response mediator protein-1 (CRMP-1), also known as dihydropyrimidinase related protein-1 (DRP-1) is a brain-specific protein that belongs to the Unc-33-related protein family (5-7). The dysregulation of cytoplasmic collapsin response mediator protein 1 (CRMP1) has been reported in brain, lung and pituitary tumors, and in EC (8-11). In prolactin-secreting pituitary adenoma, CRMP1 has been shown to be associated with tumor progression (12). The downregulation of CRMP1 has been shown to be significantly associated with advanced disease, metastasis and shorter survival in non-small cell lung cancer (NSCLC), suggesting that CRMP1 acts as a novel tumor suppressor gene $(13,14)$. Functional studies have demonstrated that the depletion of CRMP1 promotes tumor invasion, whereas its increased expression has an opposite effect in glioblastoma (9). The expression level of CRMP1 has been shown to significantly correlate with the depth of invasion and lymph node metastasis in EC (11). However, its role in EC has not yet been fully elucidated.

MicroRNAs (miRNAs or miRs) are a class of conservative single-stranded non-coding RNAs, composed of 17-25 ribonucleotides (15). The deregulation of miRNA expression was previously detected in EC (16). It was reported that the most markedly upregulated miRNAs were hsa-miR-15a, hsa-miR-28-3p, hsa-miR-31, hsa-miR-99b, hsa-miR-101, hsa-miR-130a, hsa-miR-143, hsa-miR-196b, hsa-miR-200a-3p, hsa-miR-210, hsa-miR-452 and hsa-miR-27a, whereas the most markedly downregulated miRNAs included hsa-miR-30b, hsa-miR-223, hsa-miR-454, hsa-miR-486, hsa-miR-574-3p and hsa-miR-126 in EC (16). miRNAs are also involved in numerous physiological processes of cell regulation, including differentiation, proliferation, migration, invasion, apoptosis and metastasis (17-20).

In this study, we aimed to elucidate the role and regulatory mechanisms of CRMP1 in EC. We examined the effects of miR-200a-3p on EC. As we found that CRMP1 was a direct target of miR-200a-3p, we also examined the effects of CRMP1 on EC. Our data provide evidence that miR-200a-3p promotes the proliferation of EC cells by post-transcriptionally regulating CRMP1. 


\section{Materials and methods}

Patients and samples. Human tissue samples (tumor tissue and adjacent normal tissues; $n=6$ ) were collected from patients who underwent surgical resection at People's Hospital of Henan Province, Zhengzhou, China between 2013 and 2014. All experiments were performed following the approval of the Medical Ethics Committee of the People's Hospital of Henan Province (approval no. HN141026) and after obtaining written informed consent from all patient donors.

Cell culture, plasmids and pre-miR-200a-3p/control miR, and cell transfection. The EC cell lines, TE1, TE2, TE3, TE4, TE5 and TE6, were purchased from the American Type Culture Collection (ATCC, Manassas, VA, USA and cultured in Dulbecco's modified Eagle's medium (DMEM) supplemented with $10 \%$ fetal bovine serum and $0.01 \%$ penicillin/streptomycine in a $5 \% \mathrm{CO}_{2}$ environment at $37^{\circ} \mathrm{C}$. The CRMP1-expressing plasmid/empty vector (pcDNA3.1) and the shCRMP1 plasmid/scramble plasmid were purchased from R\&D Systems (Abingdon, UK). Pre-miR-200a-3p and control-miR were purchased from Ambion (Austin, TX, USA). For transfection experiments, the cells were cultured in serumfree medium without antibiotics at $60 \%$ confluence for $24 \mathrm{~h}$. The TE2 cells were transfected with the CRMP1-expressing plasmid or empty vector, or with the shCRMP1 plasmid or scramble control plasmid for $48 \mathrm{~h}$ using Lipofectamine 2000 (Invitrogen, Carlsbad, CA, USA) according to manufacturer's instructions. In addition, the cells were transfected with pre-miR-200a-3p or control-miR. Following incubation for $6 \mathrm{~h}$, the medium was removed and replaced with normal culture medium for $48 \mathrm{~h}$, unless otherwise specified. The transfection efficiency was determined by western blot analysis.

Western blot analysis. Western blot analysis of different samples was performed to validate proteomic quantification. Protein was isolated from the 6 pairs of EC tissues and adjacent normal tissues (patient nos. 1-6) and from the 6EC cell lines. Total protein lysate was prepared using RIPA buffer containing protease inhibitors and phosphatase inhibitors. Protein lysates were resolved on a sodium dodecyl sulfate (SDS)-polyacrylamide gel and electrotransferred onhto PVDF membranes. After blocking, the membranes were immunoblotted with the following primary antibodies: anti-CRMP1 (ab199722), antip21 (ab109520), anti-CDK4 (ab108357) and anti-p53 (ab1431) (Abcam, Cambridge, MA, USA) overnight at $4^{\circ} \mathrm{C}$ and then immunoblotted with secondary antibody [goat anti-rabbit IgG H\&L (HRP); ab6721, Abcam] at room temperature. The signals were detected by enhanced chemiluminescence.

Reverse transcription-polymerase chain reaction (RT-PCR) and quantitative PCR ( $q P C R$ ) for CRMPl. Total RNA was extracted from the cells using RNeasy Plus mini kit (Qiagen, Valencia, CA, USA) according to the manufacturer's instructions. cDNA was synthesized using the QuantiTect reverse transcription kit (Qiagen). qPCR was carried out using the 7900HT Fast Real-Time PCR System according to the manufacturer's instructions (Applied Biosystems, Foster City, CA, USA). The primer sequences for CRMP1 were as follows: forward, 5'-CCCCAAAAGCGTGTGACAGTA-3' and reverse,
5'-GGTAGAAGGGATTTGTGCG-3'. Glyceraldehyde 3-phosphate dehydrogenase (GAPDH) was used as an internal control. The primers for GAPDH were as follows: forward, 5'-GAA GGTCGGAGTCAACGGATTTG-3'; and reverse, 5'-ATGGC ATGGACTGTGGTCATGAG-3'. PCR amplification was carried out under the following conditions: denaturation at $95^{\circ} \mathrm{C}$ for $10 \mathrm{~min}$, followed by 40 cycles of denaturation at $95^{\circ} \mathrm{C}$ for $15 \mathrm{sec}$, annealing at $60^{\circ} \mathrm{C}$ for $30 \mathrm{sec}$, and extension at $72^{\circ} \mathrm{C}$ for $1 \mathrm{~min}$. The relative expression level for target genes was normalized by the $\mathrm{Ct}$ value of GAPDH using the $2^{-\Delta \Delta \mathrm{Ct}}$ relative quantification method.

qPCR for miRNA expression. The mirVana miRNA isolation kit (Ambion) was used to extract the total RNA from the cells according to manufacturer's instructions. Total RNA was used to generate cDNA using the specific RT-primer from the TaqMan gene expression assay kit (Applied Biosystems). qPCR was performed to determine the expression levels of miRNA. U6 was used as an internal control. The primer sequences were as follows: miR-200a-3p, 5'-UAACACUGUCUGGUAACGAUGU-3'; and U6, 5-'CGCTTCACGAATTTGCGTGTCAT-3'.

3-(4,5-Dimethylthiazol-2-yl)-2,5-diphenyltetrazolium bromide (MTT) assay. The effects on cell proliferation were assessed by MTT assay (Sigma, St. Louis, MO, USA) which was performed as previously described (21). In brief, the cells were plated in 96-well plates in DMEM containing $10 \%$ fetal bovine serum at a density of $8 \times 10^{3}$ cells per well at $37^{\circ} \mathrm{C}$ in a $5 \% \mathrm{CO}_{2}$ incubator for $12 \mathrm{~h}$. The cells were transfected with the plasmids. MTT $(5 \mathrm{mg} /$ ml) was then added to the wells (20 $\mu \mathrm{l}$ per well). The plates were then incubated in a cell incubator for $4 \mathrm{~h}$, and the supernatant was then removed and $150 \mu \mathrm{l}$ of dimethyl sulfoxide was added to each well. Following incubation for $10 \mathrm{~min}$, the absorbance of each well was measured using a Synergy ${ }^{\mathrm{TM}} 4$ (BioTek Instruments, Winooski, VT, USA) at a wavelength of $570 \mathrm{~nm}$, with the reference wavelength set at $630 \mathrm{~nm}$. The absorbance was directly proportional to the number of surviving cells.

In vitro proliferation assay. Cell proliferation was also assessed using a colorimetric BrdU proliferation kit according to the manufacturer's instructions (cat no. 74299; Roche, Indianapolis, IN, USA). The cells were incubated with BrdU for $3 \mathrm{~h}$ and wased with phosphate-buffered saline (PBS). The genomic DNA was fixed in $4 \%$ paraformaldehyde, immunolabeled for BrdU, and counterstained with the fluorescent nuclear marker, DAPI (Sigma).

Cell cycle analysis. The cells $\left(8.0 \times 10^{5}\right.$ cells) were seeded into a $100-\mathrm{mm}$ culture plate and allowed to attach overnight. The cells were transfected with the plasmids for $24 \mathrm{~h}$, washed twice with $\mathrm{NaCl} / \mathrm{Pi}$, and then centrifuged at $200 \mathrm{x}$ g at room temperature. The pellet was resuspended in $1 \mathrm{ml}$ cold $\mathrm{NaCl} / \mathrm{Pi}$ and fixed in $70 \%$ ethanol for at least $12 \mathrm{~h}$ at $4^{\circ} \mathrm{C}$. The fixed cells were incubated with $100 \mu \mathrm{l}$ DNase-free RNase A $(200 \mu \mathrm{g} / \mathrm{ml})$ for $30 \mathrm{~min}$ at $37^{\circ} \mathrm{C}$, and then $1 \mathrm{mg} / \mathrm{ml}$ propidium iodide was added. The stained cells were analyzed using a fluorescence-activated cell sorter (BD Accuri C6; BD Biosciences, Ann Arbor, MI, USA). The percentages of cells in the G1, S and G2/M phases of the cell cycle were determined using CellQuest Pro software (FlowJo, Ashland, OR, USA). 


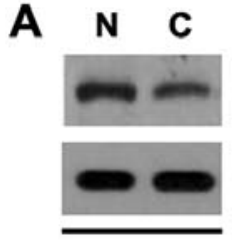

p1

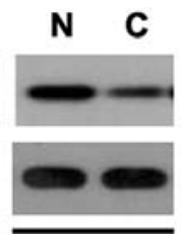

p2

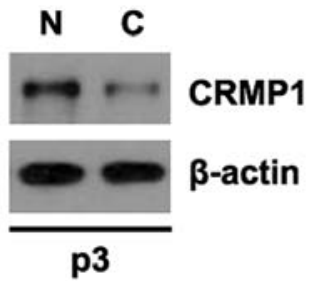

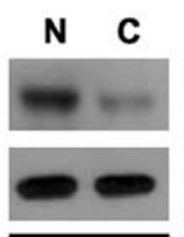

p4

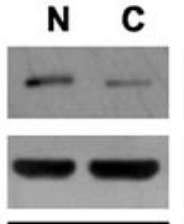

p5

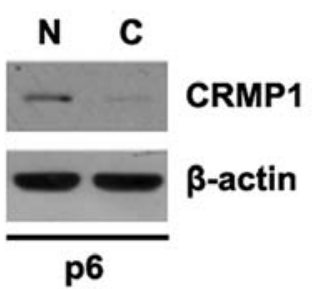

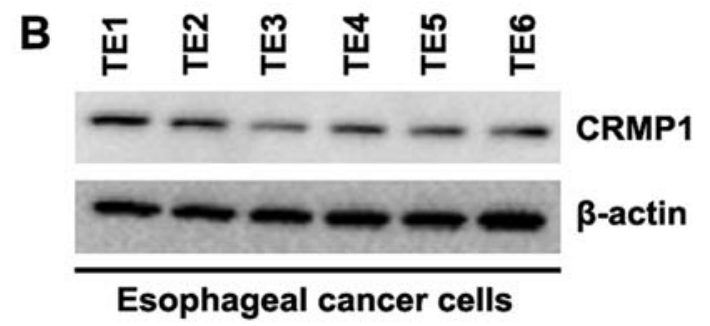

Figure 1. Cytoplasmic collapsin response mediator protein 1 (CRMP1) is downregulated in esophageal cancer and CRMP1 expression differs between different esophageal cancer cell lines. (A) Western blot analysis of CRMP1 protein expression in esophageal cancer tissues (indicted by the letter C) and adjacent normal tissues (indicted by the letter N). $\beta$-actin was used as a loading control. $n=6$ samples. (B) Western blot analysis of CRMP1 protein expression in esophageal cancer cell lines. $\beta$-actin was used a loading control. $n=3$ experiments.

Bioinformatics analysis. The analysis of potential miRNA target sites was carried out using the commonly used prediction algorithm, TargetScan (http://www.targetscan.org).

Immunofluorescence staining. The cells were fixed with paraformaldehyde before blocking with BSA. The cells were then incubated with the rabbit antibody against CRMP1 (1:200 dilution; Abcam) overnight. After washing 3 times with PBS, the cells were incubated with the secondary antibody [goat anti-rabbit IgG H\&L (HRP); ab6721, Abcam]. The labeled cells were detected under a laser scanning confocal microscope (Olympus, Tokyo, Japan).

Statistics analysis. Data are presented as the means \pm SE, and are the product of 3 independent experiments. Significance among the groups was determined using a Student's t-test. Statistical significance was set at $\mathrm{P}<0.05$.

\section{Results}

CRMP1 is downregulated in EC and CRMP1 expression differs between different $E C$ cell lines. In an aim to identify differences in CRMP1 expression between EC tissues and adjacent normal tissues, we performed western blot analysis. Protein was isolated from 6 pairs of EC tissues and normal tissues (patient nos. 1-6). We found that CRMP1 protein expression was significantly decreased in the tumor tissues, compared with the adjacent normal tissues (Fig. 1A). This suggested that CRMP1 may be a tumor suppressor gene in EC. In order to examine CRMP1 protein expression among different EC cell lines, we performed western blot analysis using 6 EC cell lines (TE1, TE2, TE3, TE4, TE5 and TE6 cells). Protein isolated from the 6 EC cell lines was examined and the results revealed that CRMP1 expression differed betwee the different EC cells (Fig. 1B). Specifically, CRMP1 expression was lowest in the TE3 cells and highest in the TE1 cells. However, for our further experiments, we selected the TE2 cells.

CRMP1 inhibits the proliferation of TE2 EC cells. To examine whether CRMP1 affects the proliferation of EC cells, firstly, by using western blot analysis, we examined whether a CRMP1expressing plasmid can be used to stably express CRMP1 protein in TE2 cells. The results revealed that CRMP1 protein expression was significantly increased by transfection of the cells with the CRMP1-expressing plasmid (Fig. 2A). In addition, we performed MTT assay to examine the proliferation of TE2 cells transfected with the CRMP1-expressing plasmid. The results revealed that the overexpression of CRMP1 inhibited the proliferation of TE2 cells after $48 \mathrm{~h}$ of transfection (Fig. 2B). To examine the effects of CRMP1 on cell proliferation, we also performed cell cycle analysis to examine its effects on the cell cycle. The results revealed lower $\mathrm{S}$ phase and $\mathrm{G} 2$ phase fractions in the TE2 cells transfected with the CRMP1-overexpressing plamid than in the cells transfected with the empty vector (Fig. 2C). To identify whether DNA synthesis inhibition contributes to the lower $\mathrm{S}$ phase fractions in the TE2 cells transfected with the CRMP1-overexpressing plamid, we performed a BrdU incorporation assay to detect DNA synthesis in the cells. The results confirmed that CRMP1 significantly inhibited DNA synthesis in the cells (Fig. 2D). In addition, we also performed western blot analysis to further confirm that CRMP1 affects proliferation markers. The results of western blot analysis demonstrated that CDK4 expression was downregulated and the expression of p53 and p21 was upregulated by the overexpression of CRMP1 (Fig. 2E).

Silencing of CRMP1 promotes the proliferation in of TE2 EC cells. In an attempt to further identify the role of CRMP1 in regulating proliferation of TE2 cells, the cells were transfected with a shCRMP1 plasmid. Following stable transfection, CRMP1 protein expression was detected by western blot anal- 

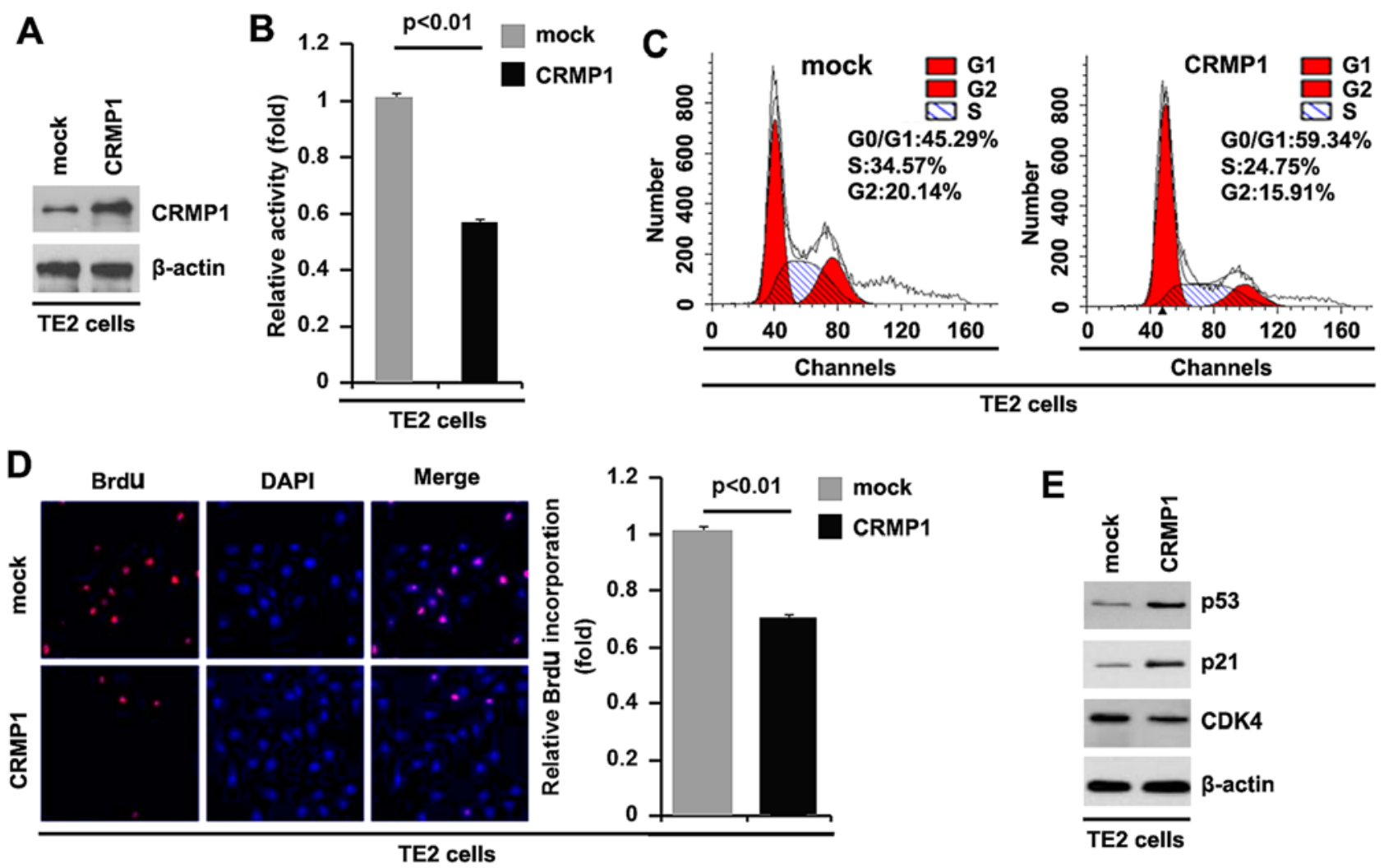

Figure 2. Cytoplasmic collapsin response mediator protein 1 (CRMP1) inhibits the proliferation of TE2 esophageal cancer cells. (A) Western blot analysis of CRMP1 protein expression in TE2 cells. TE2 cells were transfected with a CRMP1-expressing plasmid or empty vector (mock). $\beta$-actin was used as a loading control. $n=3$ experiments. (B) MTT assay of TE2 cells. TE2 cells were transfected with CRMP1-expressing plasmid or empty vector (mock) and then cell proliferation was measured at the indicated time points by MTT assay. $n=3$ experiments. (C) Cell cycle analysis of TE2 cells transfected with CRMP1-expressing plasmid or empty vector (mock). Histograms of DNA contents obtained by FACS analysis are shown. The percentages of each cell cycle stages are shown in the inset of the histograms. $n=3$ experiments. (D) BrdU incorporation assay of TE2 cells. Cells were transfected with CRMP1-expressing plasmid or empty vector (mock). $\mathrm{n}=3$ experiments. (E) Western blot analysis of CDK4, p21 and p53 protein expression in TE2 cells transfected with CRMP1-expressing plasmid or empty vector (mock). $\beta$-actin was used as a loading control. $n=3$ experiments.

ysis. The results revealed that transfection with the shCRMP1 plasmid evidently suppressed CRMP1 protein expression in the TE2 cells (Fig. 3A). Moreover, the proliferation rates of the TE2 cells were examined by MTT assay. The results revealed that the silencing of CRMP1 promoted the proliferation of the TE2 cells (Fig. 3B). This was confirmed by BrdU incorporation assay, which indicated that transfection with the shCRMP1 plasmid resulted in increased DNA synthesis activity per viable cell in the cells (Fig. 3C). To further confirm that CRMP1 regulates the proliferation of TE2 cells, we performed western blot analysis to detect the expression of proliferation markers (CDK4, p53 and p21). The results demonstrated that CDK4 expression was upregulated, and that of p53 and p21 was downregulated in the cells transfected with the shCRMP1 plasmid (Fig. 3D). The above-mentioned findings demonstrated that the silencing of CRMP1 promoted the proliferation of TE2 cells.

miR-200a-3p suppresses CRMP1 protein expression in TE2 EC cells. miRNAs/miRs are a class of non-coding RNAs able to regulate gene expression at the post-transcriptional level, by binding to the 3'UTR of target messenger RNAs (mRNAs) through partial sequence homology, and causing a block of translation and/or mRNA degradation (22-24). The upregulation of specific miRNAs can contribute to the downregulation of tumor suppressor genes (25-27). Thus, we hypothesized that CRMP1 is downregulated by miRNAs in EC.

We screened for miRNAs targeting CRMP1 using TargetScan (http://www.targetscan.org/), and a large number of target miRNAs were found. However, we were interested in miR-200a-3p, as it has been previously shown to be upregulated in EC (16). The target sites on the 3'UTR of CRMP1 are shown in Fig. 4A. We hypothesized that miR-200a-3p downregulates CRMP1 expression by targeting its $3^{\prime} \mathrm{UTR}$ in EC cells.

In order to determine whether CRMP1 is downregulated by miR-200a-3p in EC, we transfected the TE2 EC cells with pre-miR-200a-3p and qPCR was then performed to detect miR-200a-3p expression in the cells. The results revealed that transfection with pre-miR-200a-3p significantly upregulated miR-200a-3p expression (Fig. 4B). To determine whether CRMP1 protein expression is affected by miR-200a-3p, we performed immunofluorescence analyses. The results of immunofluorescence analyses revealed that CRMP1 protein expression was significantly downregulated by transfection with pre-miR-200a-3p in the TE2 cells (Fig. 4C). Moreover, western blot analysis was performed to detect CRMP1 protein expression in TE2 cells transfected with pre-miR-200a-3p. Consistent with the results of immunofluorescence analyses, we found that CRMP1 protein expression was significantly 

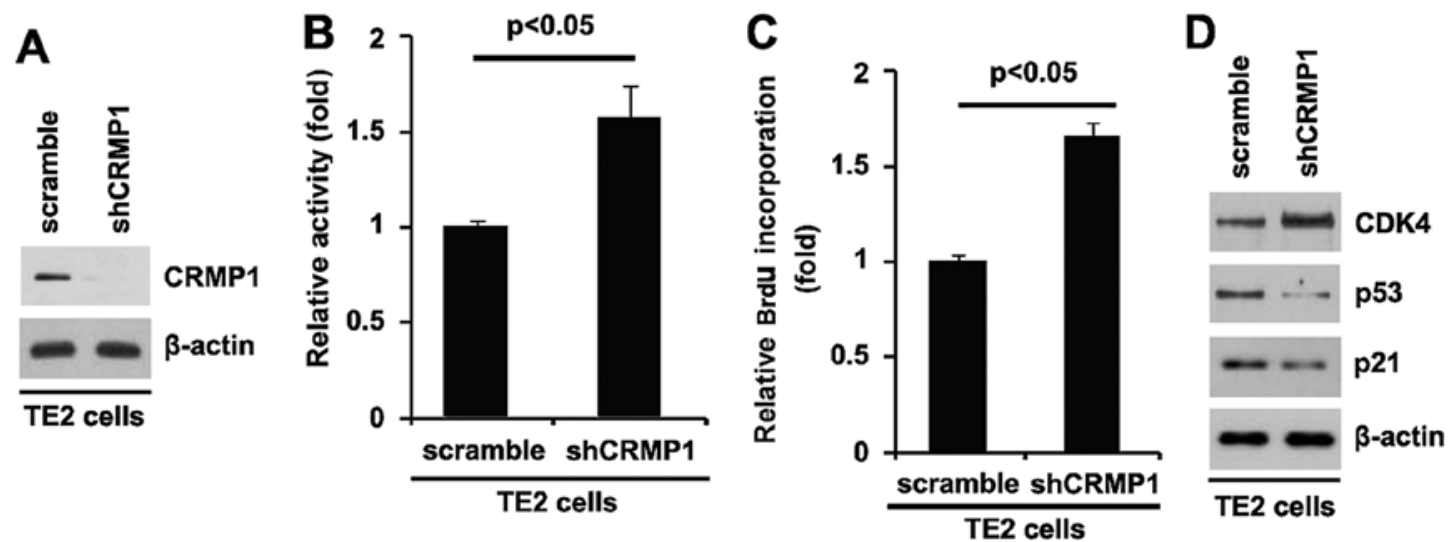

Figure 3. Knockdown of cytoplasmic collapsin response mediator protein 1 (CRMP1) promotes the proliferation of TE2 cells. (A) Western blot analysis of CRMP1 protein expression in TE2 cells. Cells were transfected with shCRMP1 plasmid or scramble control. $\beta$-actin was used as a loading control. $n=3$ experiments. (B) MTT assay of TE2 cells transfected with shCRMP1 plasmid or scramble control. $n=3$ experiments. (C) BrdU incorporation assay of TE2 cells transfected with shCRMP1 plasmid or scramble control. $n=3$ experiments. (D) Western blot analysis of CDK4, p21 and p53 protein expression in TE2 cells transfected with shCRMP1 plasmid or scramble control. $\beta$-actin was used as a loading control. $n=3$ experiments.

downregulated by transfection with pre-miR-200a-3p in TE2 cells (Fig. 4D). To determine whether CRMP1 mRNA expression was decreased by miR-200a-3p, we then performed RT-PCR and qPCR to detect CRMP1 mRNA expression in TE2 cells transfected with pre-miR-200a-3p or control $\mathrm{miR}$. The results of RT-PCR and qPCR demonstrated that miR-200a-3p did not affect CRMP1 mRNA expression in TE2 cells (Fig. 4E and F).

miR-200a-3p promotes the proliferation of TE2 EC cells. In an attempt to identify the role of miR-200a-3p in regulating the proliferation of TE2 cells, the cells were transfected with pre-miR-200a-3p. Following transfection, the proliferation rates of TE2 cells were examined by MTT assay. The results revealed that transfection with miR-200a-3p promoted the proliferation of TE2 cells (Fig. 5A). This was further revealed by BrdU incorporation assay which indicated that transfection with pre-miR-200a-3p resulted in increased DNA synthesis activity per viable cell (Fig. 5B). To further confirm that miR-200a-3p can regulate EC cell proliferation, we performed western blot analysis to detect the expression of proliferation markers (CDK4, p53 and p21). The results revealed that CDK4 expression was upregulated, and that of p53 and p21 was downregulated in the cells transfected with pre-miR-200a-3p (Fig. 5C). The above-mentioned findings demonstrated that miR-200a-3p promoted the proliferation of TE2 cells.

\section{Discussion}

EC is one of the six most common malignancies worldwide and is associated with a 5 -year survival rate $>25 \%$ (1). Understanding the molecular biology of the disease is a prerequisite to predicting prognosis and to selecting effective treatment options. The development of EC is a multistep phenomenon involving genetic events that result in key abnormalities of cell cycle regulation, growth factor activity and intercellular adhesion mechanisms (28).
Consistent with previous findings demonstrating that CRMP1 significantly inhibited the proliferation of medulloblastoma cells (29), we found that its overexpression also inhibited the proliferation of EC cells. It has been demonstrated that CRMP1 significantly inhibits the migration, invasion of and the formation of filopodia and intense stress fibers in medulloblastoma cells (29). In addition, the expression level of CRMP1 has been shown to significantly correlate with the depth of invasion and lymph node metastasis in EC (11). In the future, we aim to determine whether CRMP1 affects the migration and invasion of TE2 EC cells.

The p53 tumor suppressor lies at a nexus of cellular pathways that sense DNA damage, cellular stress and improper mitogenic stimulation (30). p53 integrates such signals and, in response, induces growth arrest, promotes apoptosis, blocks angiogenesis, or mediates DNA repair in a context-dependent manner (31). The importance of p53 in preventing tumor formation is indicated by the presence of mutations in the p53 pathway in almost all types of cancer (32). In this study, we found that CRMP1 overexpression significantly upregulated $\mathrm{p} 53$ protein expression and the silencing of CRMP1 downregulated p53 protein expression in TE2 EC cells. p21-mediated growth inhibition has been attributed to two main activities that depend on two non-overlapping structural domains: the carboxy-terminal PCNA-binding domain and the amino-terminal CDK-cyclin inhibitory domain $(33,34)$. By binding to PCNA, p21 competes for PCNA binding with DNA polymerase- $\delta$ and several other proteins involved in DNA synthesis, thus directly inhibiting DNA synthesis (35). We demonstrated that CRMP1 overexpression significantly upregulated $\mathrm{p} 21$ protein expression and the silencing of CRMP1 downregulated 21 protein expression in TE2 EC cells. Our results suggeswt that CRMP1 functions as a tumor suppressor gene by regulating p21 and p53 in EC.

As recently demonstrated through miRNA microarray analysis, miR-200 is upregulated in nasopharyngeal carcinoma (NPC). It was also shown that the endogenous miR-200a-3p expression level increases with the degree of differentiation in a panel of NPC cell lines, and that the 
A

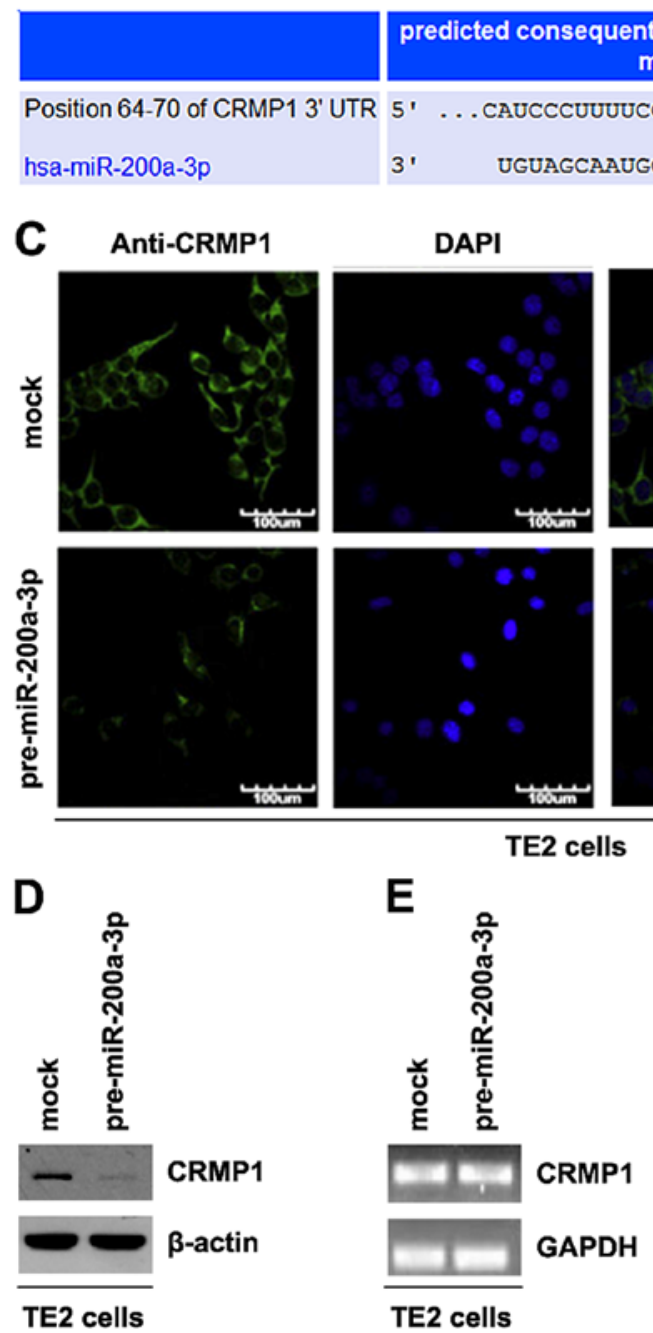

B

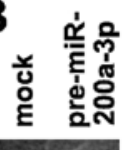

miR-200a-3p

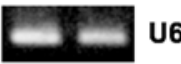

TE2 cells

mock

pre-miR-200a-3p

p $<0.01$

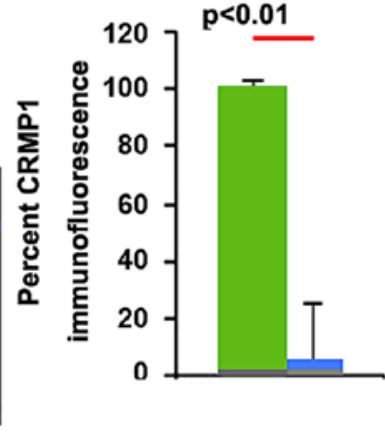

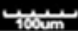

F

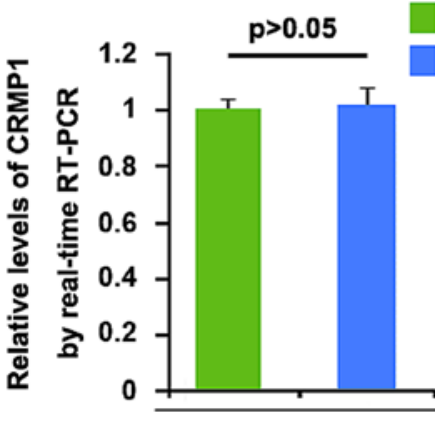

TE2 cells

Figure 4. miR-200a-3p suppresses cytoplasmic collapsin response mediator protein 1 (CRMP1) protein expression in TE2 esophageal cancer cells. (A) Schematic of predicted miR-200a-3p-binding sites in the 3'UTR of CRMP1 mRNA by TargetScan. (B) qPCR of miR-200a-3p in TE2 cells transfected with pre-miR-200a-3p or control miR (mock). U6 was used as a loading control. n=3 experiments. (C) Immunofluorescence analysis of TE2 cells transfected with pre-miR-200a-3p and control miR (mock). Left panel shows microscopic images of immunofluorescence staining of one representative experiment (magnification, x100). Right panel shows graphic presentation of mean fluorescence intensities. $n=3$ experiments. (D) Western blot analysis of CRMP1 protein expression in TE2 cells infected as indicated. $\beta$-actin was used as a loading control. $n=3$ experiments. (E) RT-PCR of CRMP1 in TE2 cells. TE2 cells were infected with pre-miR-200a-3p or control miR (mock). Glyceraldehyde 3-phosphate dehydrogenase (GAPDH) was used as a loading control. n=3. (F) qPCR of CRMP1 in TE2 cells. TE2 cells were infected with pre-miR-200a-3p or control miR (mock). GAPDH was used as a loading control. $n=3$ experiments.
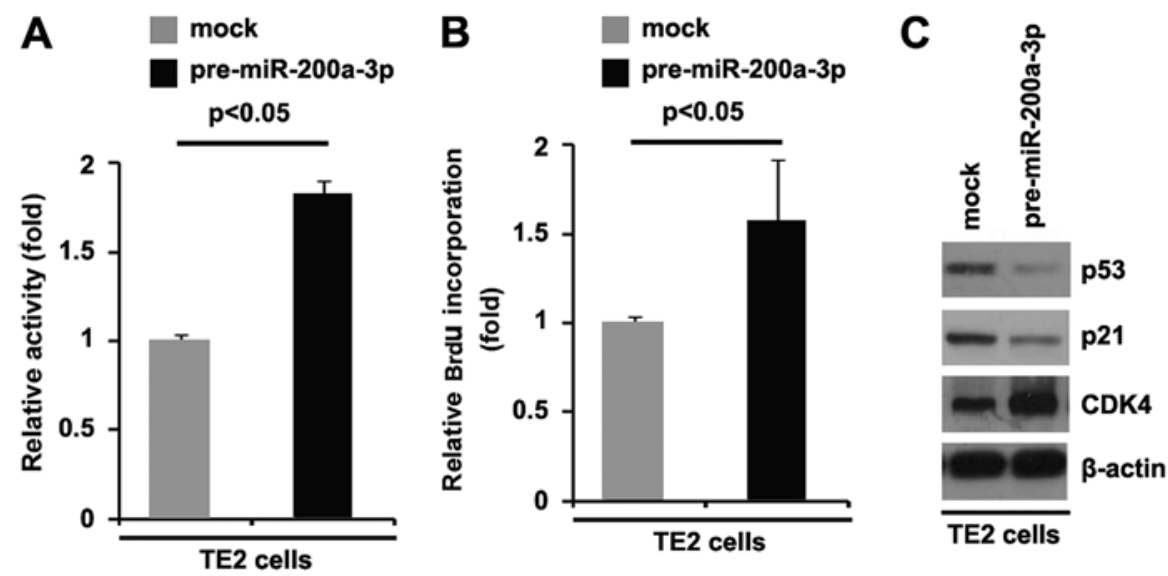

Figure 5. pre-miR-200a-3p promotes the proliferation of TE2 cells. (A) MTT assay of TE2 cells transfected with pre-miR-200a-3p or control miR (mock). $\mathrm{n}=3$ experiments. (B) BrdUJ incorporation analysis for TE2 cells transfected with pre-miR-200a-3p or control miR (mock). $\mathrm{n}=3$ experiments. (C) Western blot analysis of CDK4, p21 and p53 protein expression in TE2 cells transfected with pre-miR-200a-3p or control miR (mock). $\beta$-actin was used as a loading control. $\mathrm{n}=3$ experiments. 
overexpression of miR-200a-3p inhibits C666-1 cell growth, migration and invasion, whereas its knockdown stimulates these processes in CNE-1 cells (36). However, the role of miR-200a-3p remains unclear in EC. In this study, we demonstrated that miR-200a-3p post-transcriptionally regulates CRMP1 and stimulates the proliferation of human EC cells.

The recognition of the differential regulation and function of CRMP1 in EC will ultimately provide a better understanding of the signaling pathways that can be therapeutically modulated. We have merely just begun to explore the role of miR-200a-3p in EC.

\section{References}

1. Enzinger PC and Mayer RJ: Esophageal cancer. N Engl J Med 349: 2241-2252, 2003

2. Ries LAG, Eisner MP, Kosary C, Hankey BF, Miller BA, Clegg LX and Edwards BK (eds): SEER Cancer Statistics Review, 1973-1999. National Cancer Institute, Bethesda, MD 2002.

3. Daly JM, Fry WA, Little AG, Winchester DP, McKee RF, Stewart AK and Fremgen AM: Esophageal cancer: Results of an American College of Surgeons Patient Care Evaluation Study. J Am Coll Surg 190: 562-573, 2000.

4. Pisani P, Parkin DM, Bray F and Ferlay J: Erratum: Estimates of the worldwide mortality from 25 cancers in 1990 . Int J Cancer 83 18-29, 1999.

5. Goshima Y, Nakamura F, Strittmatter P and Strittmatter SM Collapsin-induced growth cone collapse mediated by an intracellular protein related to UNC-33. Nature 376: 509-514, 1995.

6. Li W, Herman RK and Shaw JE: Analysis of the Caenorhabditis elegans axonal guidance and outgrowth gene unc-33. Genetics 132: 675-689, 1992

7. Hamajima N, Matsuda K, Sakata S, Tamaki N, Sasaki M and Nonaka M: A novel gene family defined by human dihydropyrimidinase and three related proteins with differential tissue distribution. Gene 180: 157-163, 1996.

8. Gao M, Yeh PY, Lu YS, Chang WC, Kuo ML and Cheng AL: NF-kappaB p50 promotes tumor cell invasion through negative regulation of invasion suppressor gene CRMP-1 in human lung adenocarcinoma cells. Biochem Biophys Res Commun 376 283-287, 2008

9. Mukherjee J, DeSouza LV, Micallef J, Karim Z, Croul S, Siu KW and Guha A: Loss of collapsin response mediator Protein1, as detected by iTRAQ analysis, promotes invasion of human gliomas expressing mutant EGFRvIII. Cancer Res 69: 8545-8554, 2009.

10. Wierinckx A, Auger C, Devauchelle P, Reynaud A, Chevallier P, Jan M, Perrin G, Fèvre-Montange M, Rey C, Figarella-Branger D et al: A diagnostic marker set for invasion, proliferation, and aggressiveness of prolactin pituitary tumors. Endocr Relat Cancer 14: 887-900, 2007.

11. Zhai J, Wang Y, Yang F, Hu J, Qi Q and Zhang Y: DRP-1, ezrin and E-cadherin expression and the association with esophageal squamous cell carcinoma. Oncol Lett 8: 133-138, 2014.

12. Raverot G, Wierinckx A, Dantony E, Auger C, Chapas G, Villeneuve L, Brue T, Figarella-Branger D, Roy P, Jouanneau E, et al; HYPOPRONOS: Prognostic factors in prolactin pituitary tumors: Clinical, histological, and molecular data from a series of 94 patients with a long postoperative follow-up. J Clin Endocrinol Metab 95: 1708-1716, 2010.

13. Shih JY, Yang SC, Hong TM, Yuan A, Chen JJ, Yu CJ, Chang YL, Lee YC, Peck K, Wu CW, et al: Collapsin response mediator protein- 1 and the invasion and metastasis of cancer cells. J Natl Cancer Inst 93: 1392-1400, 2001.

14. Shih JY, Lee YC, Yang SC, Hong TM, Huang CY and Yang PC: Collapsin response mediator protein-1: A novel invasionsuppressor gene. Clin Exp Metastasis 20: 69-76, 2003.

15. Cho WC: OncomiRs: The discovery and progress of microRNAs in cancers. Mol Cancer 6: 60,2007.
16. Liu SG, Qin XG, Zhao BS, Qi B, Yao WJ, Wang TY, Li HC and Wu XN: Differential expression of miRNAs in esophageal cancer tissue. Oncol Lett 5: 1639-1642, 2013.

17. Lee KH, Goan YG, Hsiao M, Lee CH, Jian SH, Lin JT, Chen YL and Lu PJ: MicroRNA-373 (miR-373) post-transcriptionally regulates large tumor suppressor, homolog 2 (LATS2) and stimulates proliferation in human esophageal cancer. Exp Cell Res 315: 2529-2538, 2009.

18. Tian Y, Luo A, Cai Y, Su Q, Ding F, Chen H and Liu Z: MicroRNA-10b promotes migration and invasion through KLF4 in human esophageal cancer cell lines. J Biol Chem 285 7986-7994, 2010

19. Kong KL, Kwong DL, Chan TH, Law SY, Chen L, Li Y, Qin YR and Guan XY: MicroRNA-375 inhibits tumour growth and metastasis in oesophageal squamous cell carcinoma through repressing insulin-like growth factor 1 receptor. Gut 61: 33-42, 2012.

20. Imanaka Y, Tsuchiya S, Sato F, Shimada Y, Shimizu K and Tsujimoto G: MicroRNA-141 confers resistance to cisplatininduced apoptosis by targeting YAP1 in human esophageal squamous cell carcinoma. J Hum Genet 56: 270-276, 2011.

21. Xiang Y, Lu DL, Li JP, Yu CX, Zheng DL, Huang X, Wang ZY, $\mathrm{Hu}$ P, Liao XH and Zhang TC: Myocardin inhibits estrogen receptor alpha-mediated proliferation of human breast cancer MCF-7 cells via regulating MicroRNA expression. IUBMB Life 68: 477-487, 2016.

22. Lee RC, Feinbaum RL and Ambros V: The C. elegans heterochronic gene lin-4 encodes small RNAs with antisense complementarity to lin-14. Cell 75: 843-854, 1993.

23. Pasquinelli AE, Reinhart BJ, Slack F, Martindale MQ, Kuroda MI, Maller B, Hayward DC, Ball EE, Degnan B, Müller P, et al: Conservation of the sequence and temporal expression of let-7 heterochronic regulatory RNA. Nature 408: 86-89, 2000.

24. Reinhart BJ, Slack FJ, Basson M, Pasquinelli AE, Bettinger JC, Rougvie AE, Horvitz HR and Ruvkun G: The 21-nucleotide let-7 RNA regulates developmental timing in Caenorhabditis elegans. Nature 403: 901-906, 2000.

25. Meng F, Henson R, Wehbe-Janek H, Ghoshal K, Jacob ST and Patel T: MicroRNA-21 regulates expression of the PTEN tumor suppressor gene in human hepatocellular cancer. Gastroenterology 133: 647-658, 2007.

26. Zhu S, Wu H, Wu F, Nie D, Sheng S and Mo YY: MicroRNA-21 targets tumor suppressor genes in invasion and metastasis. Cell Res 18: 350-359, 2008.

27. Zhu S, Si ML, Wu H and Mo YY: MicroRNA-21 targets the tumor suppressor gene tropomyosin 1 (TPM1). J Biol Chem 282: 14328-14336, 2007.

28. Tselepis C, Perry I and Jankowski J: Barrett's esophagus: Disregulation of cell cycling and intercellular adhesion in the metaplasia-dysplasia-carcinoma sequence. Digestion 61: 1-5, 2000.

29. Li KK, Qi Y, Xia T, Yao Y, Zhou L, Lau KM and Ng HK: CRMP1 inhibits proliferation of medulloblastoma and is regulated by HMGA1. PLoS One 10: e0127910, 2015.

30. Levine AJ, Hu W and Feng Z: The P53 pathway: What questions remain to be explored? Cell Death Differ 13: 1027-1036, 2006.

31. Ko LJ and Prives C: p53: puzzle and paradigm. Genes Dev 10: 1054-1072, 1996

32. Hollstein M, Sidransky D, Vogelstein B and Harris CC: p53 mutations in human cancers. Science 253: 49-53, 1991.

33. Chen J, Jackson PK, Kirschner MW and Dutta A: Separate domains of p21 involved in the inhibition of Cdk kinase and PCNA. Nature 374: 386-388, 1995.

34. Luo Y, Hurwitz J and Massagué J: Cell-cycle inhibition by independent CDK and PCNA binding domains in p21Cip1. Nature 375: 159-161, 1995.

35. Moldovan GL, Pfander B and Jentsch S: PCNA, the maestro of the replication fork. Cell 129: 665-679, 2007.

36. Xia H, Ng SS, Jiang S, Cheung WK, Sze J, Bian XW, Kung HF and Lin MC: miR-200a-mediated downregulation of ZEB2 and CTNNB1 differentially inhibits nasopharyngeal carcinoma cell growth, migration and invasion. Biochem Biophys Res Commun 391: 535-541, 2010. 